\title{
Statistical evaluation of dosimetric differences changes between the Modified Batho's density correction method and the Anisotropic Analytical Algorithm for clinical practice
}

\author{
Abdulhamid Chaikh1,2, Jacques Balosso',2 \\ Department of Radiation Oncology \& Medical Physics, University Hospital of Grenoble, Grenoble, France \\ University Grenoble-Alpes - Grenoble, France
}

Received December 22, 2015; Revised June 20, 2016; Accepted June 20, 2016; Published Online June 30, 2016

\section{Original Article}

\begin{abstract}
Purpose: The aim of this work was to assess and to quantify, for clinical practice, the differences in computed doses using two types of dose calculation algorithm for the heterogeneity correction including target volumes and organs at risk (OARs). Methods: 35 patients having lung, breast, spine, head \& neck, brain and pelvic tumors, were studied. For each patient, 2 treatment plans were generated. In plan 1 , the dose was calculated using the Modified Batho's (MB) density correction method integrated in the Pencil Beam Convolution algorithm. In plan 2, the dose was calculated using the Anisotropic Analytical Algorithm (AAA). To compare the two plans a dosimetric analysis was carried out including cumulative and differential dose volume histograms (DVH), coverage index, and conformity index. Wilcoxon signed rank and Spearman's tests were used to calculate $p$-values and correlation coefficients (r), respectively. Bootstrap simulation with 1000 random samplings was used to calculate the $95 \%$ confidence interval $(95 \% \mathrm{CI})$. Results: The analysis of DVH showed that the AAA method calculated significantly higher doses for OARs for all cancer sites and lower doses for target volumes, especially for targets located in lung, with $\mathrm{p}<0.05$. The data demonstrated a strong correlation between MB and AAA for all cancer sites with $r>0.9$. Conclusion: This study confirms that using the AAA integrated into Eclipse ${ }^{\circledR}$ TPS, the calculated dose will be increased to OARs, and reduced to target volumes. Thus, when changing from the MB algorithm to AAA, attention should be paid to avoid any bias of over/under estimating the dose given by AAA and to hold discussions between physicists and oncologists regarding any necessary modification in the prescription method.
\end{abstract}

Keywords: Heterogeneity correction; Modified Batho's density correction; AAA; Medical decision.

\section{Introduction}

Recent advances in radiotherapy have introduced several new dose calculation algorithms to accurately compute the prescribed dose according to the variable density of heterogeneous tissues.

The former algorithms used a correction factor to take account of the heterogeneity of tissues, e.g. density correction methods are integrated into the Pencil Beam Convolution algorithm (PBC) in the Eclipse ${ }^{\circledR}$ Treatment Planning System (TPS) (Varian Medical Systems, Palo Alto, CA), such as the Modified Batho's (MB) density correction method. In these algorithms, the alteration in the lateral transport of electrons is not modelled. ${ }^{1-5}$ Presently, numerous algorithms approximate the transport of electrons, such as the Anisotropic Analytical Algorithm (AAA) and Collapsed Cone Convolution (CCC) implemented, respectively, in the Eclipse ${ }^{\circledR}$ and Pinnacle ${ }^{\circledR}$ TPS (Philips Radiation Oncology Systems, Fitchburg, WI). More recently, Acuros XB (Varian Medical Systems, Inc., Palo Alto, CA) solves the linear Boltzmann transport equation. In contrast to AAA, the Acuros XB algorithm calculates dose to a medium, which can be converted into the dose to water for treatment plan evaluation. Several studies have demonstrated that AAA and Acuros 
XB algorithms were more accurate for the calculation of dose within heterogeneous media.[6-9] However, we have little knowledge about the impact of heterogeneity corrections on target volume and organs at risk using the AAA algorithms in clinical practice. Recently, numerous studies, recommended that a sufficient number of cases for each anatomical site should be used to evaluate the dose difference resulting from the former and the new algorithms ${ }^{9}$. In the present study, we assess and evaluate what the impact would be, when switching from $\mathrm{MB}$ to $\mathrm{AAA}$, on doses for six cancer sites exhibiting lower or higher tissue densities.

\section{Methods and Materials}

\subsection{Clinical cases and treatment planning}

This study is based on 35 patients and includes six different cancer types. These cases were chosen to cover the full range of tumors treated in radiation oncology, namely: lung, breast, spine, head \& neck, brain and pelvis. A computed tomography (CT-scan) was carried out for each patient. Then the images were loaded into the TPS. The virtual simulation for each patient was generated by a digitally reconstructed radiograph and beam's eye view information. The planning target volumes (PTVs) including the security margins, and the organs at risk (OARs) were delineated by the radiation oncologist. Table 1 shows the tumor locations, the number of PTVs, the prescribed dose and the treatment fields for all cases.

For each patient, two treatment plans were generated. In plan 1, the dose was calculated using the MB method in combination with the PBC algorithm; this was taken as the reference dose. In plan 2, the dose was calculated using AAA. The reference treatment plans were designed according to the clinical experience of the specialist department and ICRU recommendations. These reference plans were validated by a medical physicist regarding two criteria: i) that $95 \%$ of the prescribed dose encompassed the PTV and the maximum dose within the PTV was under $107 \%$ of the prescribed dose; ii) for OARs, that the recommended dose constraints were respected. Table 2 shows the dose constraints considered for the OARs in all cancer cases. The choice of plan 1 as the reference plan is based on current clinical results which have been obtained over many years of clinical experience.

\subsection{Treatment plan evaluations 2.2.1 Dosimetric analysis} Dose Volume Histogram (DVH): for each PTV, the minimum dose (Dmin), mean dose (Dmean), maximum dose (Dmax) and the calculated dose to $95 \%$ of the target volume (D95\%) were compared using cumulative cDVH. The dose constraints for each OAR, presented in Table 2 were compared.

Quality indices: the Coverage Index (CI) and Conformity Index for the target volume (CITV) proposed by the Radiation Therapy Oncology Group (RTOG) were compared using the following equations ${ }^{10,11}$ :

$$
\mathrm{CI}=\frac{\mathrm{Imin}}{\mathrm{RI}}
$$

Where Imin is the minimal isodose in gray surrounding the target and RI is the reference isodose in gray.

$$
\text { CITV }=\frac{\text { Volume receiving } 95 \% \text { of prescribed dose }}{\mathrm{TV}}
$$

where TV is the target volume.

\begin{tabular}{|c|c|c|c|c|c|}
\hline Cancer sites & $\begin{array}{c}\text { PTV cc } \\
\text { average } \pm \sigma\end{array}$ & $\begin{array}{l}\text { Prescribed dose [Gy] } \\
\text { average [min-max] }\end{array}$ & Techniques & $\begin{array}{l}\text { Energy } \\
{[\mathrm{MV}]}\end{array}$ & Total fields \\
\hline $\begin{array}{l}\text { Lung } \\
(n=6)\end{array}$ & $394 \pm 194$ & $58.8[50.8-66]$ & 3DRT & 18 & 34 \\
\hline $\begin{array}{l}\text { Breast } \\
(n=6)\end{array}$ & $1059 \pm 248$ & $47.2[40-50.6]$ & 3DRT & 6 and 18 & 38 \\
\hline $\begin{array}{l}\text { Spine } \\
(n=6)\end{array}$ & $465.4 \pm 221.6$ & $10[8-20]$ & 3DRT & 6 and 18 & 19 \\
\hline $\begin{array}{l}\text { Head \& neck } \\
\qquad(n=6)\end{array}$ & $228.2 \pm 135.9$ & $56.9[44.0-69.9]$ & IMRT & 6 & 34 \\
\hline $\begin{array}{l}\text { Brain } \\
(n=5)\end{array}$ & $318.2 \pm 339.1$ & $57[54-66]$ & IMRT & 6 & 25 \\
\hline $\begin{array}{l}\text { Pelvis } \\
(\mathrm{n}=6)\end{array}$ & $276.7 \pm 249.3$ & $65.3[52.7-76]$ & IMRT & 6 & 42 \\
\hline
\end{tabular}

Table 1: Tumor locations, the PTV, the prescribed dose and the treatment fields for all patients. 
Table 2: Dose constraints for OARs in all cancer cases.

\begin{tabular}{|c|c|c|}
\hline Cancer sites & OARs & Dose constraints \\
\hline \multirow{4}{*}{ Lung } & Lung (minus the PTV) & V20 Gy <30\% \\
\hline & & V30 Gy $<20 \%$ \\
\hline & Spinal cord $+2 \mathrm{~mm}$ & Dmax $<45$ Gy \\
\hline & Heart & V40 Gy $<30 \%$ \\
\hline \multirow[t]{3}{*}{ Breast } & Lung & V20 Gy <30\% \\
\hline & & V30 Gy $<20 \%$ \\
\hline & Heart & V40 Gy $<30 \%$ \\
\hline \multirow[t]{2}{*}{ Spine } & Spinal cord & Dmax $<45$ Gy \\
\hline & Kidney & Dmean < 18 Gy \\
\hline \multirow[t]{4}{*}{ Head \& neck } & Spinal cord & Dmax $<45$ Gy \\
\hline & Parotid & Dmean < 24 Gy \\
\hline & Larynx & Dmean $<50$ Gy \\
\hline & Oral cavity & Dmean $<30$ Gy \\
\hline \multirow[t]{4}{*}{ Brain } & Brain stem & Dmax $<55$ Gy \\
\hline & Cochlea & Dmax $<54$ Gy \\
\hline & Optic nerves & Dmax $<55$ Gy \\
\hline & Optic chiasm & Dmax $<54$ Gy \\
\hline \multirow[t]{5}{*}{ Pelvis } & Bladder & V60 Gy $<50 \%$ \\
\hline & & Dmax $<78$ Gy \\
\hline & Rectum & V60 Gy $<50 \%$ \\
\hline & & Dmax $<74$ Gy \\
\hline & Femoral head & V50Gy $<10 \%$ \\
\hline
\end{tabular}

The dose homogeneity inside the PTV were compared using the S-index associated with the differential dDVH 12:

$$
S-\text { index }=\sqrt{\frac{\sum_{\mathrm{j}=1}^{\mathrm{TV}}(\mathrm{D}(\mathrm{j})-\mathrm{Dmean})^{2}}{\mathrm{TV}}}
$$

where $D(j)$ is the relative dose in voxel $j$ of the lesion, Dmean is the average relative dose in the lesion and TV is the target volume in elementary voxels.

\subsubsection{Statistical analysis}

For each patient, to compare plan 2 with plan 1, the difference in percentage was calculated as:

$$
\Delta \text { Dose }(\%)=\left(D_{A A A}-D_{M B}\right) \times 100 / D_{A A A}
$$

Positives values indicate that the calculated dose using plan 2 with AAA was higher than the calculated using plan 1 with $\mathrm{MB}\left(\mathrm{D}_{\mathrm{AAA}}>\mathrm{D}_{\mathrm{MB}}\right)$ and negative values mean the opposite $\left(\mathrm{D}_{\mathrm{AAA}}<\mathrm{D}_{\mathrm{MB}}\right)$.

The Wilcoxon signed rank test was used to calculate the p-value. A bilateral statistical test was realized with an error $\alpha=5 \%$, corresponding to a $95 \%$ confidence interval $(95 \% \mathrm{CI})$. The dose difference is considered significant if $\mathrm{p}<0.05$. Data are presented as average $(\mu)$ \pm standard deviation (SD). Spearman's test was used to calculate the correlation coefficient (r). A bootstrap simulation method with 1000 random samplings was used to calculate the $95 \%$ confidence interval, $95 \%$ CI. ${ }^{13}$, 14

\section{Results}

\subsection{Dose volume histograms (DVH)}

Tables 3 and 4 summarize the dosimetric and statistical results for PTVs and OARs. In Table 3 , it can be seen that AAA algorithms calculated a significantly lower dose for PTVs located in the lung, with $\mathrm{p}<0.05$. In Table 4 , it appears clearly that, the dose calculated for OARs by AAA was higher than using the MB algorithm. Spearmen's test showed a strong correlation with $r>0.9$ for all dosimetric parameters. Figure 1 shows cDVH calculated using MB for plan 1 and AAA for plan 2 .

\subsection{Quality indices}

Figure 2 shows the $S$-index values associated with dDVH, using a boxplot representation. It can be seen in Figure 2 that MB calculated more homogenous dose distribution than AAA with $p<0.05$ and $r>0.9$. Figure 3 shows the indice values using a boxplot representation. 
Tables 3: The dosimetric and statistical results for PTVs. The average $(\mu)$ presents the dose difference in $\%$ and SD presents the standard deviation.

\begin{tabular}{|c|c|c|c|c|c|}
\hline Cancer sites & Dose & $\bar{\mu} \mu \pm \mathrm{SD}$ & $95 \% \mathrm{CI}$ & r-value & p-value \\
\hline \multirow[t]{4}{*}{ Lung } & Dmin & $-8.7 \pm 4.6$ & {$[-10.1 ;-7.3]$} & 0.94 & 0.01 \\
\hline & Dmean & $-1.8 \pm 1.2$ & {$[-2.2 ;-1.5]$} & 0.98 & 0.01 \\
\hline & D95\% & $-6.8 \pm 4.5$ & {$[-8.2 ;-5.5]$} & 0.93 & 0.007 \\
\hline & Dmax & $0.3 \pm 2.6$ & {$[-0.5 ; 1.0]$} & 0.97 & 0.7 \\
\hline \multirow[t]{4}{*}{ Breast } & Dmin & $11.4 \pm 17.6$ & {$[2.9 ; 20]$} & 0.97 & 0.008 \\
\hline & Dmean & $0.43 \pm 0.67$ & {$[0.1 ; 0.8]$} & 0.99 & 0.03 \\
\hline & D95\% & $5.2 \pm 9.4$ & {$[0.4 ; 9.9]$} & 0.99 & 0.01 \\
\hline & Dmax & $6.7 \pm 23.1$ & {$[-5.3 ; 18.0]$} & 0.70 & 0.02 \\
\hline \multirow[t]{4}{*}{ Spine } & Dmin & $9.2 \pm 13.6$ & {$[3.7 ; 22.2]$} & 0.91 & 0.03 \\
\hline & Dmean & $-1.7 \pm 4.6$ & {$[-6.1 ; 2.6]$} & 0.99 & 1.0 \\
\hline & D95\% & $2.8 \pm 3.6$ & {$[-0.5 ; 6.2]$} & 0.99 & 0.15 \\
\hline & Dmax & $1.6 \pm 4.9$ & {$[-3.1 ; 6.3]$} & 0.99 & 0.83 \\
\hline \multirow{4}{*}{$\begin{array}{c}\text { Head \& } \\
\text { neck }\end{array}$} & Dmin & $-0.9 \pm 1.6$ & {$[-1.4 ; 0.4]$} & 0.99 & 0.12 \\
\hline & Dmean & $0.66 \pm 2.3$ & {$[0.2 ; 1.2]$} & 0.98 & 0.44 \\
\hline & D95\% & $-0.59 \pm 0.88$ & {$[-0.8 ;-0.4]$} & 0.99 & 0.1 \\
\hline & Dmax & $0.65 \pm 0.57$ & {$[0.5 ; 0.8]$} & 0.99 & 0.004 \\
\hline \multirow[t]{4}{*}{ Brain } & Dmin & $-0.12 \pm 0.9$ & {$[-0.4 ; 0.2]$} & 0.99 & 1.0 \\
\hline & Dmean & $0.04 \pm 0.13$ & {$[0.01 ; 0.1]$} & 0.99 & 0.67 \\
\hline & D95\% & $-0.1 \pm 0.6$ & {$[-0.3 ; 0.1]$} & 0.99 & 0.87 \\
\hline & Dmax & $0.11 \pm 0.3$ & {$[0.1 ; 0.2]$} & 0.99 & 0.5 \\
\hline \multirow[t]{4}{*}{ Pelvis } & Dmin & $-0.02 \pm 1.3$ & {$[-0.2 ; 0.2]$} & 0.99 & 0.75 \\
\hline & Dmean & $0.18 \pm 0.62$ & {$[0.1 ; 0.3]$} & 0.99 & 0.5 \\
\hline & D95\% & $0.12 \pm 1.0$ & {$[-0.1 ; 0.3]$} & 0.99 & 0.5 \\
\hline & Dmax & $0.09 \pm 0.4$ & {$[0.1 ; 0.2]$} & 0.99 & 0.78 \\
\hline
\end{tabular}
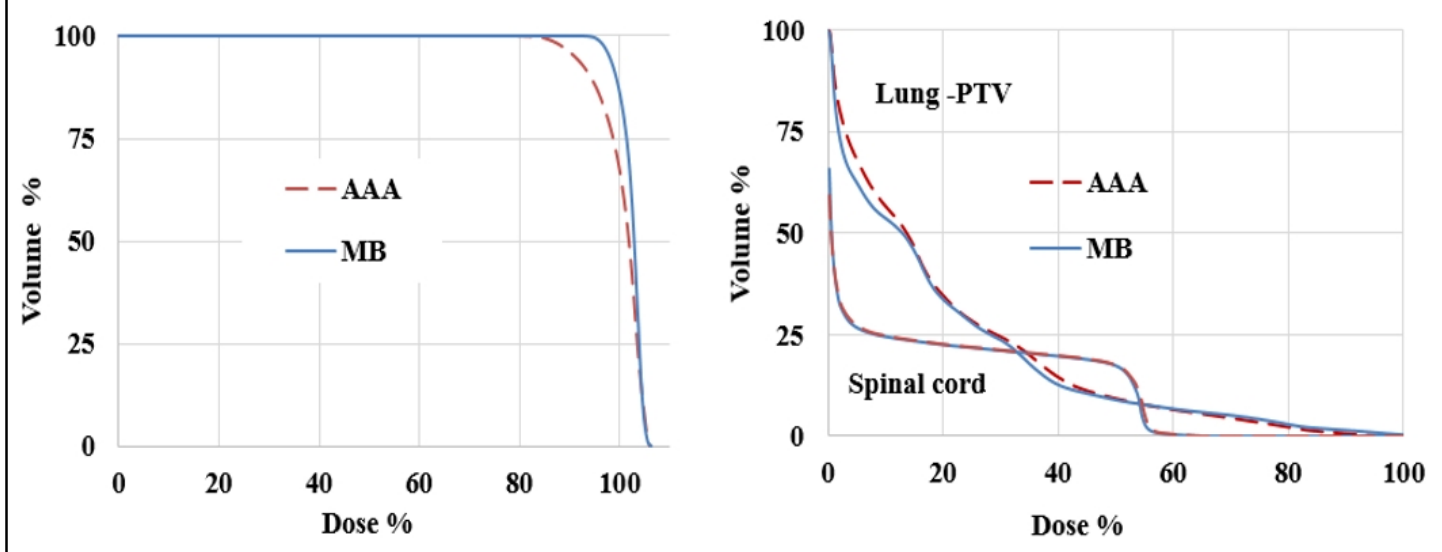

Figure 1: Cumulative dose volume histograms for lung calculated using the MB method with 1D heterogeneity correction for plan 1 and AAA for plan 2. 
Tables 4: The dosimetric and statistical results for OARs. The average $(\mu)$ presents the dose difference in $\%$ and SD presents

\begin{tabular}{|c|c|c|c|c|c|c|}
\hline Cancer sites & OARs & Dose & $\mu \pm S D$ & $95 \% \mathrm{CI}$ & r-value & p-value \\
\hline \multirow[t]{3}{*}{ Lung } & $\begin{array}{r}\text { Lung (minus the } \\
\text { PTV) }\end{array}$ & $\begin{array}{l}\text { V20 Gy } \\
\text { V30 Gy } \\
\text { Dmean }\end{array}$ & $\begin{array}{c}8.1 \pm 4.0 \\
1.3 \pm 6.1 \\
1.9 \pm\end{array}$ & $\begin{array}{c}4.1 ; 12.1] \\
{[-4.6 ; 7.3]} \\
{[-2.8 ; 6.7]}\end{array}$ & $\begin{array}{l}0.99 \\
0.97 \\
0.90\end{array}$ & $\begin{array}{c}0.03 \\
0.6 \\
0.4\end{array}$ \\
\hline & Spinal cord & Dmax & $0.8 \pm 1.0$ & {$[0.1 ; 1.8]$} & 0.98 & 0.1 \\
\hline & Heart & V40 Gy & $1.4 \pm 3.2$ & {$[-1.6 ; 4.5]$} & 0.99 & 0.3 \\
\hline \multirow[t]{2}{*}{ Breast } & Lung & $\begin{array}{l}\text { V20 Gy } \\
\text { V30 Gy } \\
\text { Dmean }\end{array}$ & $\begin{array}{c}10.7 \pm 10.3 \\
12.4 \pm 12.0 \\
14.4 \pm 8.0\end{array}$ & $\begin{array}{l}{[0.8 ; 20.8]} \\
{[1.1 ; 23.6]} \\
{[6.5 ; 22.3]}\end{array}$ & $\begin{array}{l}0.99 \\
0.99 \\
0.99\end{array}$ & $\begin{array}{l}0.06 \\
0.03 \\
0.03\end{array}$ \\
\hline & Heart & Dmax & $19.2 \pm 13.3$ & {$[6.9 ; 31.5]$} & 0.99 & 0.03 \\
\hline \multirow[t]{2}{*}{ Spine } & Spinal cord & Dmax & $0.8 \pm 0.6$ & {$[0.2 ; 1.3]$} & 0.99 & 0.03 \\
\hline & Kidney & Dmean & $2.0 \pm 1.0$ & {$[0.1 ; 4.0]$} & 0.99 & 0.8 \\
\hline \multirow[t]{4}{*}{ Head \& neck } & Spinal cord & Dmax & $3.1 \pm 1.5$ & {$[1.7 ; 4.5]$} & 0.99 & 0.03 \\
\hline & Parotid & Dmean & $4.3 \pm 4.4$ & {$[0.8 ; 6.0]$} & 0.99 & 0.1 \\
\hline & Larynx & Dmean & $9.1 \pm 15$ & {$[0.1 ; 4.7]$} & 0.99 & 0.1 \\
\hline & Oral cavity & Dmean & $-0.97 \pm 7.6$ & {$[-8.4 ; 6.6]$} & 0.99 & 0.1 \\
\hline \multirow[t]{4}{*}{ Brain } & Trunk & Dmax & $1.12 \pm 1.1$ & {$[0.3 ; 2.6]$} & 0.64 & 0.2 \\
\hline & Cochlea & Dmax & $3.7 \pm 2.7$ & {$[1.8 ; 5.6]$} & 0.98 & 0.01 \\
\hline & Optic nerves & Dmax & $2.4 \pm 4.9$ & {$[1.8 ; 5.8]$} & 0.99 & 0.3 \\
\hline & Optic chiasm & Dmax & 1.12 .9 & {$[-2.2 ; 4.3]$} & 0.98 & 0.8 \\
\hline \multirow[t]{3}{*}{ Pelvis } & Bladder & $\begin{array}{l}\text { V60 Gy } \\
\text { Dmax }\end{array}$ & $\begin{array}{l}3.3 \pm 3.7 \\
0.5 \pm 0.5\end{array}$ & $\begin{array}{l}{[0.0 ; 6.9]} \\
{[-0.3 ; 2.6]}\end{array}$ & $\begin{array}{l}0.99 \\
0.99\end{array}$ & $\begin{array}{l}0.1 \\
0.1\end{array}$ \\
\hline & Rectum & $\begin{array}{l}\text { V60 Gy } \\
\text { Dmax }\end{array}$ & $\begin{array}{c}1.5 \pm 2 \\
-0.2 \pm 0.3\end{array}$ & $\begin{array}{c}{[0.7 ; 3.7]} \\
{[-0.5 ; 0.2]}\end{array}$ & $\begin{array}{l}0.99 \\
0.99\end{array}$ & $\begin{array}{l}0.1 \\
0.1\end{array}$ \\
\hline & Femoral head & V50 Gy & $15.1 \pm 38.3$ & {$[-7.9 ; 38.1]$} & 0.92 & 0.2 \\
\hline
\end{tabular}




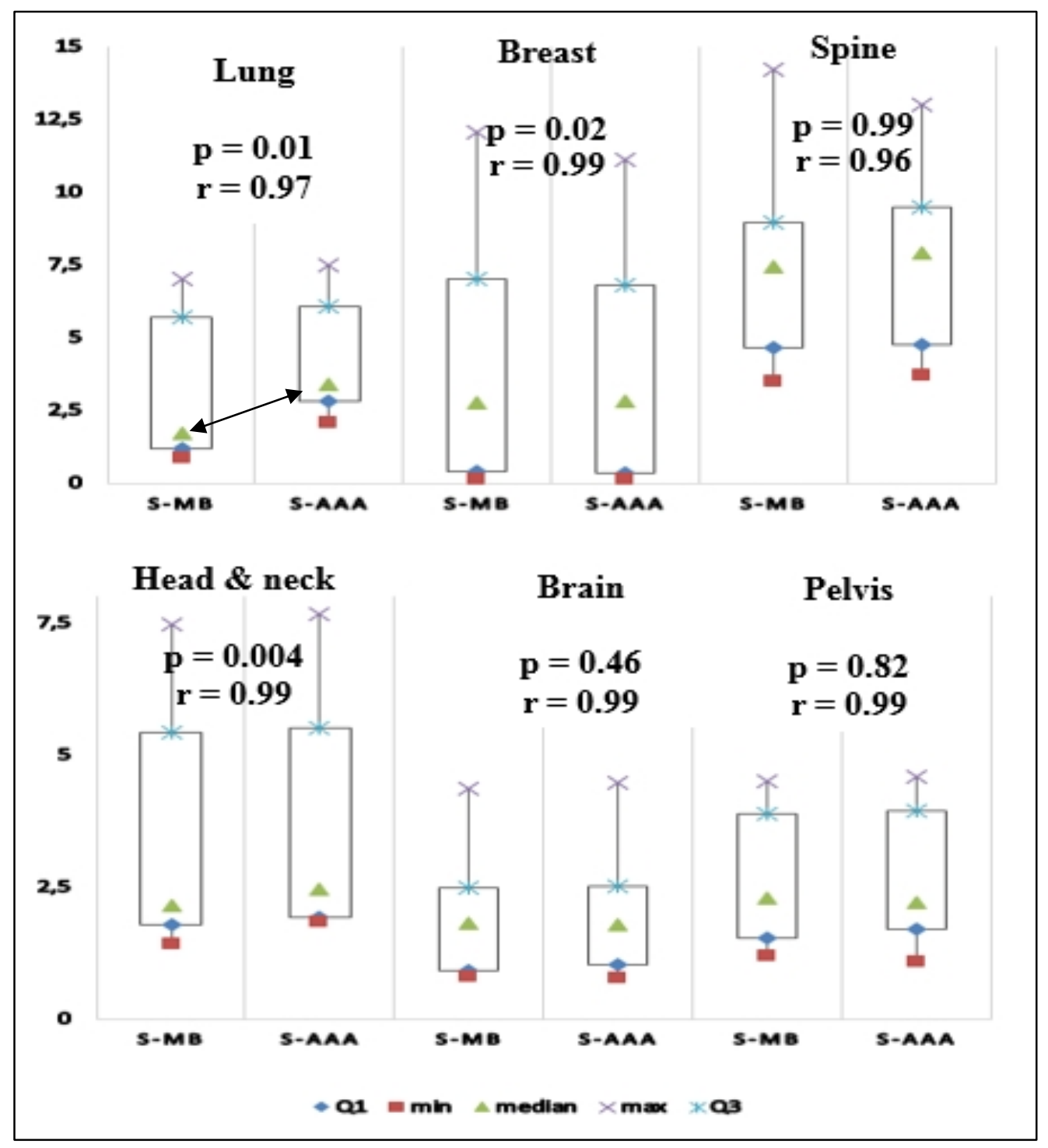

Figure 2: Boxplot for S-index obtained from MB method in plan 1 and AAA in plan 2 indicating the minimum (min), median and maximum (max) values, as well as the 25th (Q1) and 75th (Q3) percentiles. 


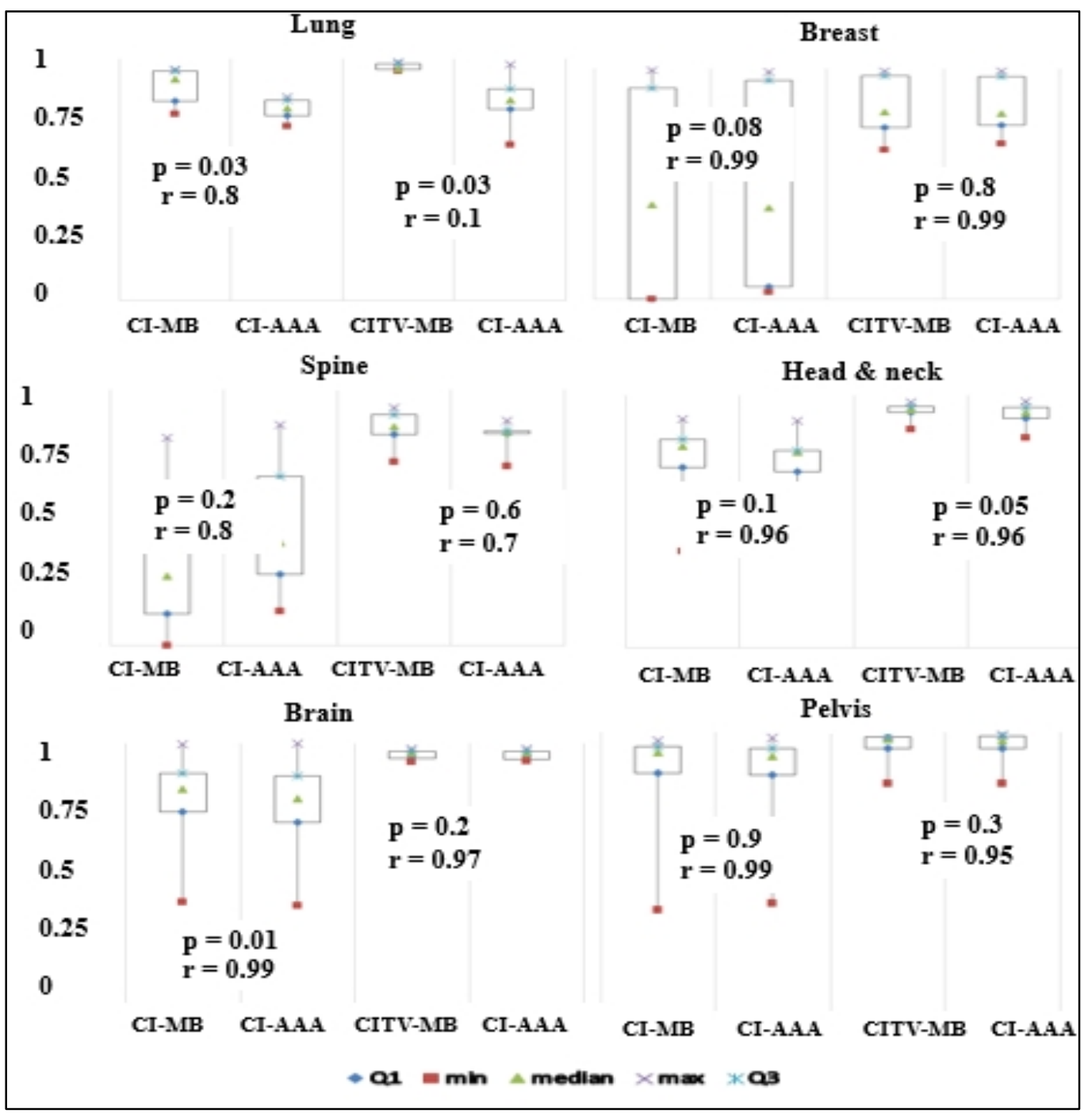

Figure 3: Boxplot for quality indices obtained from MB method in plan 1 and AAA in plan 2 indicating the minimum (min), median and maximum (max) values, as well as the 25th (Q1) and 75th (Q3) percentiles.

\section{Discussion}

Many authors have evaluated the recent density correction methods and algorithms in terms of their abilities to accurately represent the dose distribution. ${ }^{15-18}$ They observed a large impact on the dose calculation particularly for heterogeneous media. They suggested that these corrections and algorithms should be used for more accurate calculation during clinical radiotherapy planning. Recently, numerous studies, recommended using AAA or Acuros XB for calculating the dose in heterogeneous media. ${ }^{7,8,20,21,22}$

In our study, we tackled the problem of changing the calculation algorithms from MB with 1D heterogeneity correction to AAA so as to offer the clinician treatment plans that are much nearer to reality than the plans generated using the MB method. We raised the question as to whether the clinician should adapt the dose prescription when moving from MB to AAA. We present a detailed analysis of the differences between DVH and quality indices for six typical treatment cases calculated using the two different algorithms. The MB method is not very accurate as a correction method; it only takes into account the density in 1D and it does not allow for changes in lateral electron transport. Conversely, AAA models accommodate lateral electron transport. We observed that tissues with lower densities, such as lung, are subject to a significant difference between MB and AAA. The chest exhibits a highly heterogeneous anatomy with bone, lung and soft tissues. Usually treatment consists of both lateral and oblique beams, which traverse low-density lung tissue. Since AAA is more accurate in correcting for heterogeneity it showed a dosimetric impact on the PTVs and OARs. Thus, there are significant differences in the dose for PTVs and OARs for lung, breast and spine. In clinical practice, the dose distribution is not uniform over the PTVs due to heterogeneous tissue densities. Our comparison of S-indexes showed that MB tends to give a better PTV coverage with homogenous dose distribution. 
Nevertheless, the AAA method is closer to the reality because it takes into account the lateral electron transport, as mentioned above. The observed difference in dose distribution is due to the effect of lateral electrons and it is expected to be larger for tissue with lower density. This explains why AAA under estimated the dose for some areas of PTVs and hot spots in normal tissue outside the PTVs. However, the dose difference varied from one cancer site to another and from patient to patient. This means that the geometric configuration, or the dose weight of beams, must be optimized to fulfil the dose constraints for OARs.

Whatever the differences are, Chaikh et al. showed that these differences could be quantified and presented by means of a color-code using the gamma index. ${ }^{23-24}$ The gamma tool shows a visual representation of the difference in dose distribution to the patient. They reported that the only realistic dose distribution is that evaluated by the clinical results obtained to the benefit of the patient. Currently, in reality the representation of the dose distribution calculated by an algorithm has clinical value when it is linked to the clinician's experience. These results should be discussed among clinicians and medical physicists to decide whether a modification of the current prescription procedure and plan acceptability criteria should be considered.

\section{Conclusion}

This study should alert physicians to treatment modifications associated with a change from the Modified Batho's method to AAA. The heterogeneity correction algorithms are not equal from the point of view of their ability to calculate the dose, especially for lung, breast and spine cases. This is due to the fact that AAA approximates lateral electron transport, which shows a significant impact on dose distribution. The physicist should be careful when changing from older algorithms to newer algorithms, in order to maximally protect the healthy tissues and to continue to achieve an optimal clinical outcome.

\section{Conflict of interest}

The authors declare that they have no conflicts of interest. The authors alone are responsible for the content and writing of the paper.

\section{References}

1. Papanikolaou N, Battista J, Mackie T, et al. Tissue inhomogeneity corrections for megavoltage photon beams. AAPM Report No 85; Task Group No. 65, 2004.

2. Ahnesjö A, Aspradakis M M. Dose calculations for external photon beams in radiotherapy. Phys Med Biol. 1999; 44 :99-155.
3. Batho HF. Lung corrections in cobalt 60 beam therapy. J Can Assoc Radiol. 1964;15:79-83.

4. El-Khatib E, Battista J J. Improved lung dose calculation using tissue-maximum ratios in the Batho correction. Med Phys.1984;11:279-86.

5. Thomas S J. A modified power low formula for inhomogeneity corrections in beams of high energy x rays. Med Phys. 1991;18:719-23.

6. Rana S. Clinical dosimetric impact of Acuros XB and analytical anisotropic algorithm (AAA) on real lung cancer treatment plans: review. Int J Cancer Ther Oncol. 2014;2:02019.

7. Ojala J. The accuracy of the Acuros XB algorithm in external beam radiotherapy - a comprehensive review. Int J Cancer Ther Oncol. 2014;2:020417.

8. Rana S, Pokharel S. Dose-to-medium vs. dose-to-water: Dosimetric evaluation of dose reporting modes in Acuros XB for prostate, lung and breast cancer. Int J Cancer Ther Oncol. 2014;2:020421.

9. Chaikh A, Balosso J. Should the dose prescription be readjusted when using tissues density corrections algorithms for radiation oncology? J Case Rep Onc Ther. 2014; 1(1):01018.

10. Feuvret L, Noël G, Mazeron JJ, Bey J. Conformity index: a review. Int J Radiation Oncology Biol Phys. 2006; (64)2: 333-342.

11. Chaikh A, Giraud JY, Balosso J. A method to quantify and assess the dosimetric and clinical impact resulting from the heterogeneity correction in radiotherapy for lung cancer. Int J Cancer TherOncol. 2014; 2(1):020110.

12. Yoon M, Park SY, Shin D, et al. A new homogeneity index based on statistical analysis of the dose-volume histogram. Journal of Applied Clinical Medical Physics 2007, 8(2): 9-17.

13. Chaikh A, Giraud JY, Perrin E, et al. The choice of statistical methods for comparisons of dosimetric data in radiotherapy. Radiation oncology 2014; 9:205.

14. Efron B, Tibshirani RJ (1993). An introduction to the bootstrap. Chapman \& Hall, New York.

15. Gray A, Oliver LD, Johnston PN. The accuracy of the pencil beam convolution and anisotropic analytical algorithms in predicting the dose effects due to attenuation from immobilization devices and large air gaps. Med Phys. 2009; 36 (31): 81-91.

16. Rana S, Pokharel S. Verification of dose calculation algorithms in a multi-layer heterogeneous phantom using films. Gulf J Oncolog. 2013;1(14):63-9.

17. Narabayashi M, Mizowaki T, Matsuo Y, et al. Dosimetric evaluation of the impacts of different heterogeneity correction algorithms on target doses in stereotactic body radiation 
therapy for lung tumors. J Radiat Res. 2012;53(5):777-84.

18. Ahnesjö A, Aspradakis M M. Dose calculations for external photon beams in radiotherapy. Phys Med Biol. 1999; 44 : 99-155.

19. Rana S, Rogers K, Pokharel S, Cheng C. Evaluation of Acuros XB algorithm based on RTOG 0813 dosimetric criteria for SBRT lung treatment with RapidArc. J Appl Clin Med Phys. 2014;15(1):4474.

20. Lu L. Dose calculation algorithms in external beam photon radiation therapy. Int J Cancer Ther Oncol. 2013; 1(2):01025.

21. Amankwaa-Frempong E, Vernimmen F, Blay S, Ezhilalan R. Irradiation of lung and esophagus tumors: A comparison of dose distributions calculated by anisotropic analytical algorithm and pencil beam convolution algorithm, a retrospective dosimetric study. Int J Cancer Ther Oncol. 2014; 2(2):020210.

22. Chaikh A, Giraud JY, Marguet M, et al. A decision tool to adjust the prescribed dose after change in the dose calculation algorithm. Int J Cancer Ther Oncol. 2014; 2(4):020414.

23. Chaikh A, Giraud JY, Balosso J. A 3D quantitative evaluation for assessing the changes of treatment planning system and irradiation techniques in radiotherapy. Int J Cancer Ther Oncol. 2014; 2(3):02033.

24. Chaikh A, Desgranges C, Balosso J. Statistical methods to evaluate the correlation between measured and calculated dose using a quality assurance method in IMRT. Int J Cancer Ther Oncol. 2015; 3(4):3411. 